\title{
The effect of supplementation with a calcium rich marine-derived multi-mineral supplement and short-chain fructo-oligosaccharides on serum lipid concentrations in postmenopausal women: a 24 month double blind randomized controlled study
}

\author{
E. M. McSorley, B. E. Cronin, P. J. Allsopp, M. M. Slevin, P. J. Magee, M. B. E. Livingstone \\ and J.J. Strain \\ Northern Ireland Centre for Food and Health, University of Ulster, Coleraine, BT52 ISA
}

Calcium (Ca) supplements are widely taken by postmenopausal women owing their role in the maintenance of bone health and the prevention of osteoporosis. Beneficial effects on circulating high-density lipoprotein (HDL), low-density lipoprotein (LDL) and total cholesterol (TC) have also been reported following Ca supplementation with or without vitamin $\mathrm{D}^{(1)}$. Short-chain fructooligosaccharides (scFOS) increase Ca absorption in vivo and thus may mediate beneficial effects on lipid concentrations ${ }^{(2)}$. The aim of this study was to investigate the effects of a $\mathrm{Ca}$ rich marine-derived multi-mineral supplement administered alone or in combination with scFOS on serum lipid concentrations in postmenopausal women.

A total of 214 postmenopausal women (mean BMI $27.3($ SD 4.7$) \mathrm{kg} / \mathrm{m}^{2}$ ) aged 48-75 years completed a two year double-blind placebo controlled trial. Participants were randomly assigned to daily supplements of $800 \mathrm{mg}$ of Ca $\left(2 \cdot 4 \mathrm{~g} \mathrm{Aquamin}{ }^{\circledR}\right)(n=75), 800 \mathrm{mg}$ of Ca with $3 \mathrm{~g}$ of scFOSs $\left(3 \cdot 2 \mathrm{~g}\right.$ Nutraflora $\left.{ }^{\circledR}\right)(\mathrm{CaFOS})(n=60)$ or maltodextrin (MD) $(n=79)$. The TC, LDL, HDL and triglyceride concentrations were measured at baseline and 24 months. Per-protocol analysis was undertaken using ANCOVA (with baseline measures as covariates) to assess time $\times$ treatment effects between groups, controlling for age, BMI and baseline Ca intake (mean intake $868 \mathrm{mg} \mathrm{Ca}$ /day) using least significant difference for post hoc comparisons.

A significant time $\times$ treatment effect was observed for LDL for the Ca $(P=0.009)$ and CaFOS groups $(P=0 \cdot 01)$. Furthermore, a significant time $\times$ treatment effects were observed for TC for the Ca and CaFOS groups $(P=0.02$ and $P=0.03$ respectively). There was no significant treatment effect of $\mathrm{Ca}$ or CaFOS on HDL, LDL:HDL and triglycerides. Supplementation with this Ca rich marinederived multi-mineral supplement has beneficial effects on LDL and TC concentrations in postmenopausal women over two years. It is plausible that the available $\mathrm{Ca}$ is binding to fat and forming insoluble Ca-fatty soaps within the gut. These insoluble Ca-fatty soaps may limit lipid absorption and subsequently increase lipolysis ${ }^{(3)}$. Administration of Aquamin may have a role in maintaining cardiovascular health in women and further research on this area is warranted.

This work was funded by Marigot Ltd (Cork, Ireland), Ingredion Inc. (Westchester, IL) and a PhD studentship received from the Department for Employment and Learning.

1. Schnatz P, Jiang X, Vila-Wright S et al. (2014) Menopause (Epublication ahead of print version).

2. Morohashi T, Sano T, Ohta A et al. (1998) J Nutr 128, 1815-8.

3. Major G, Alarie J, Phouttama S et al. (2007) AJCN 85, 54-9. 